\title{
WebLab-Deusto-CPLD: A Practical Experience
}

\author{
http://dx.doi.org/10.3991/ijoe.v8iS1.1952
}

\author{
J. García-Zubía, I. Angulo, P. Orduna, D. López-de-Ipina, \\ U. Hernández, L. Rodríguez, O. Dziabenko, V. Canivell \\ University of Deusto, Bilbao, Spain
}

\begin{abstract}
This paper shows the experience at the University of Deusto with the WebLab-Deusto-CPLD in the subject "Programmable Logic" of the Faculty of Engineering in the field of Digital Electronics. Presented herein is a technical overview of the laboratory, and its characteristics.
\end{abstract}

Index Terms-Engineering education, Student experiments, remote laboratories.

\section{WEBLAB-DEUSTO PLATFORM ${ }^{1}$}

A remote laboratory is a tool, which provides students an access to real experiments through Internet.

At this moment in the classroom WebLab-Deusto is used with different experiments: WebLab-Deusto-CPLD, WebLab-Deusto-FPGA, WebLab-Deusto-PIC, WebLabDeusto-GPIB, WebLab-Deusto-Logic, WebLabDEUSTO-Microbot, VISIR, and LXI-VISIR. Furthermore the platform already supports new schemas based on Virtual Machines that will be used in the near future at the University. Fig. 1 shows the weblab.deusto.es page which includes demos, mobile lab, Facebook integration, and different options offered to students by WebLab-Deusto. The work described here is centered in VHDL, FPGA and CPLD of Xilinx.

WebLab-Deusto is an Open Source platform. It can be easily deployed to offer different remote experiments. It is important to distinguish between platforms that manage a number of remote experiments (rigs) and domaindependent remote experiments (i.e. "electronics remote laboratory”). MIT iLabs, LabShare Sahara [1] and WebLab-Deusto are good examples of these rigs while VISIR [2] is a good example of the domain-dependent remote experiments.

Since February 2005, WebLab-Deusto is being used in its different versions at the University of Deusto. Until September 2007, WebLab-Deusto was a domaindependent experiment platform - WebLab-Deusto v1.WebLab-Deusto v2 was based on AJAX and web services while WebLab-Deusto v3 [3] promoting the integration of new experiments in an easy way for developers (plug-and-play).

Today's WebLab-Deusto v4 is a robust platform that uses web standards suitable for mainstream web browsers, and adapts to mobile devices. It can be downloaded and deployed to serve new remote experiments in different environments and operating systems. The effort has been put to offer a secure and scalable design, using SSl in communications and allows for untrusting experiment developers in the management layers, providing tracking

\footnotetext{
${ }^{1}$ This work is part of "Formacion 2020" project and it was supported by Diputación de Bizkaia under BizkaiLab
}

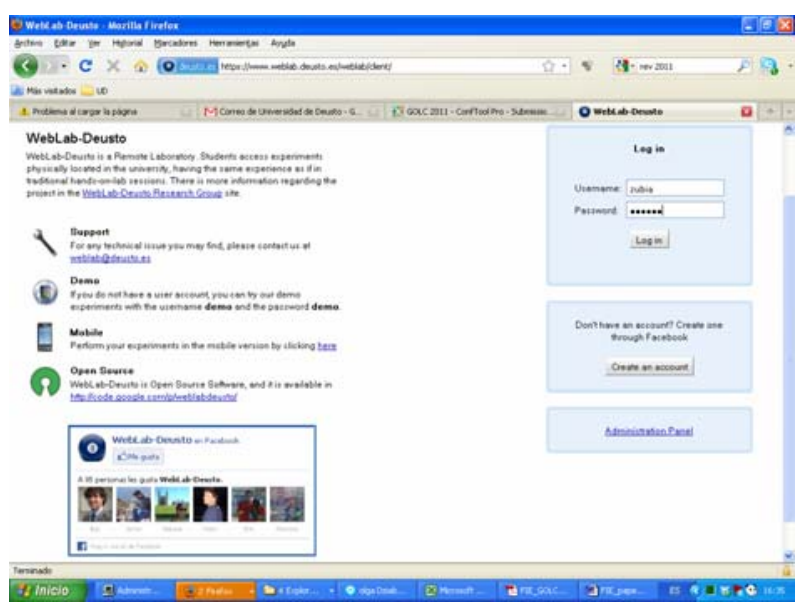

Figure 1. WebLab-DEUSTO main page and experiments

down the use of the system and the messages sent by students.

The system also supports different authentication schemes such as a regular database, LDAP, external students OpenID. It is extensible enough to be integrated in a number of platforms such as, Graaasp, Facebook, .LRN and Moodle, and to integrate external experiments such as VISIR.

In order to support a usage of hundreds of students, administration tools have been developed, which come out of the box with the available packages of WebLab-Deusto.

\section{SUBJECT AND REMOTE EXPERIMENT}

The University of Deusto is using the experiment WebLab-Deusto-CPLD together with the subject "Programmable Logic". This subject is offered for the third-year students of Electronics and Control Engineering Degree.

The course includes VHDL - VHSIC hardware description language- which is used in electronic design automation to describe digital and mixed-signal systems such as field-programmable gate arrays and integrated circuits, and CPLD -complex programmable logic device. The course is based on the Project Based Learning (PBL) approach offering the students the lab assignments and projects as main activities.

Students learn to program in VHDL, and to develop, implement and test the designs in an educational board in traditional laboratory. Weekly student's assignment is to program, and to implement a circuit design. The student decides how and on which equipment - remote or traditional labs- he will execute the assignment; by uploading a video with the design running in the remote lab or in the classical lab. The remote laboratory is not a replacement rather a complement of the traditional lab to promote the 


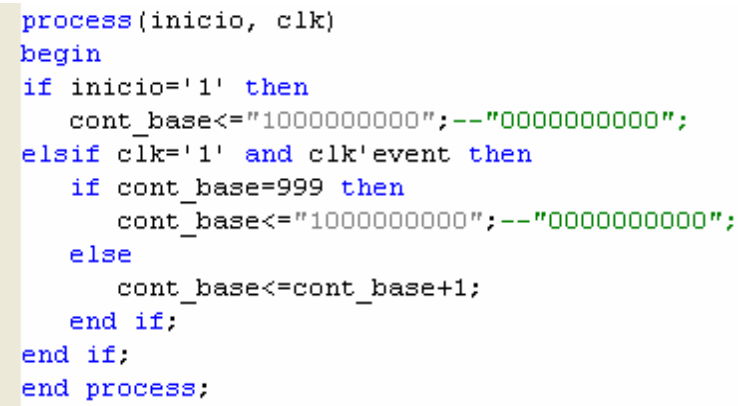

Figure 2. VHDL Code for designing

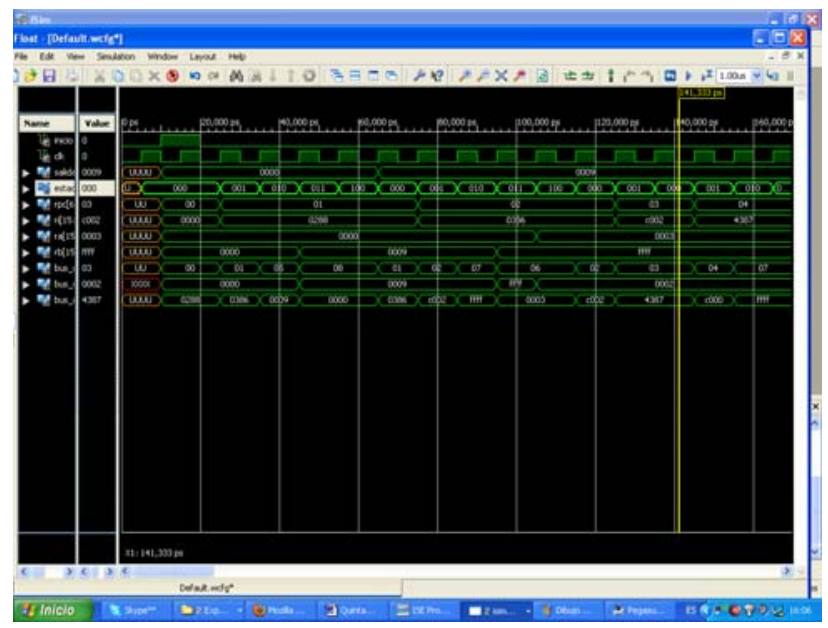

Figure 3. Simulation of a VHDL Code

\section{(-) weblabdeusto}
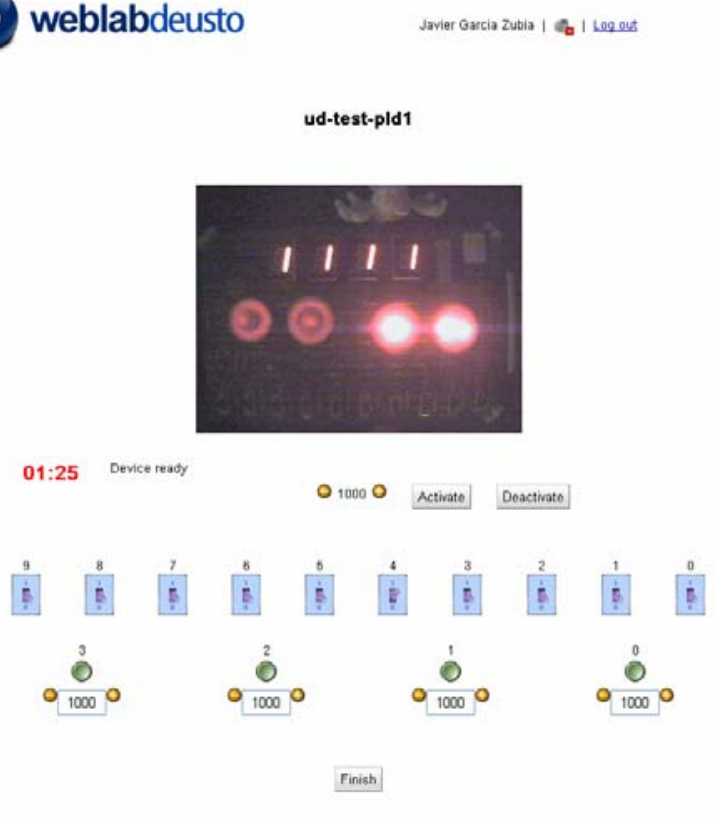

Figure 4. WebLab-DEUSTO-PLD interface autonomous and significant learning, and to support students.

In order to accomplish the assignment the student writes the VHDL code (Fig. 2), simulates it (Fig. 3) and obtains a binary file.

Student accesses the WebLab-Deusto -a demo is available- using the university authorization validated through LDAP. Once logged-in the student uploads the generated binary file. From this moment the student has control of the board during 200 seconds. The board is based in Xilinx CPLD XC9572 and its interface consists of 10 switches, 4 buttons, 1 clock, 6 LEDs, and 4 sevensegments. Then he tests the design using these inputs and sees an evolution of the system through the WebCam (see Fig. 4).

If the program is running properly, student records the results on video. Finally the student uploads the program and the video into Moodle - LMS system of the University of Deusto. The use of WebLab-Deusto is an optional tool to complete the assignment.

Administration of the access to the WebLab occurs based on a priority queue. The platform automatically shows the student the availability and his position in the queue, and surrenders the control of the experiment when the student's turn arrives.

\section{CONCLUSIONS}

The presentation of this work will be based in a real demonstration of WebLab-DEUSTO-CPLD during the conference. The experiment proposed is offered through the WebLab-DEUSTO architecture and it will be accessed with any web browser in any type of device (PC, lap-top, mobile phone or tablet) under any operating system without security problems with open ports and firewalls. These characteristics promote the deployment of this remote laboratory to other universities and institutions.

\section{REFERENCES}

[1] Kostulski, T., Murray, S., (2010). “The National Engineering Laboratory Survey”. Labshare Project. December 2010.

[2] Gustavsson et al (2009) "On objectives of instructional laboratories, individual assessment, and use of collaborative remote laboratories", IEEE Transactions on Learning Technologies, TLT, VOL. 2, No 4. (2007)

[3] Garcia-Zubia, J et al (2009). Addressing Software Impact in the Design of Remote Labs”. IEEE Transactions on Industrial Electronics (Journal). ISSN: 0278-0046; DOI: 10.1109/ TIE.2009.2026368. Volume 56, Issue 12, Dec. 2009 Page(s):47574767.

\section{AUTHORS}

J. Garcia-Zubia, I. Angulo, D. López-de-Ipina, U. Hernández, L. Rodríguez and V. Canivell are with the Faculty of Engineering of the University of Deusto, Bilbao, Spain (e-amil: zubia@deusto.es)

P. Orduna and O. Dziabenko are with DeustoTech University of Deusto, Bilbao, Spain.

This work is a description of a demonstration given during $1^{\text {st }} \mathrm{Ex}-$ periment@ International Conference, 17/18 November 2011 in Lisbon, Portugal. It was supported by Diputación de Bizkaia, Bilbao, Spain and by Calouste Gulbenkian Foundation, Lisbon, Portugal. Manuscript received 01 December 2011. Published as resubmitted by the authors 20 January 2012. 